\title{
Feasibility Analysis of Translocating Western Pearlshell Mussels into the Cherry Creek Native Trout Project Reach on the Flying D Ranch of Montana
}

A Report to Turner Enterprises, Inc.

\author{
by \\ David Stagliano
}

Montana Natural Heritage Program

Helena, Montana

April 2013
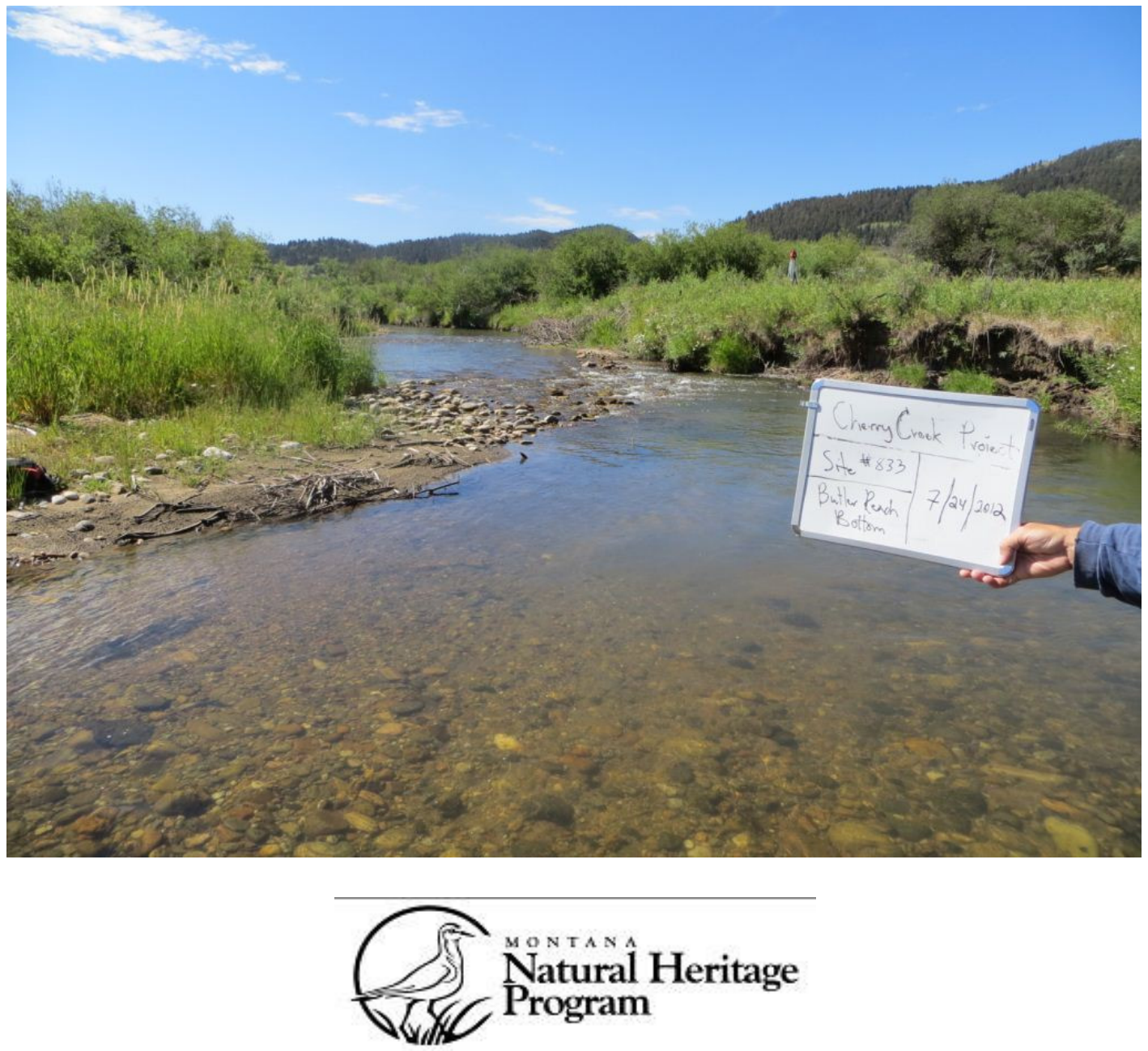

a program of the Montana State Library's Natural Resource Information System operated by the University of Montana 


\section{Introduction}

The western pearlshell mussel (WEPE), Margaritifera falcata has experienced significant range reductions across Montana in the last 100 years and is now known from $~ 88$ populations, of which, only 20 are expected to be viable 100 years from now (Stagliano 2010). Many of these remaining populations are also at risk of extirpation due to stochastic events capable of wiping out these isolated small populations, and from lack of reproduction with non-native salmonid host species. Cherry Creek below the natural canyon barrier on the Flying D Ranch is one such stream that currently has a non-viable population (D-rank, Stagliano unpublished data 2010), and closer to the confluence with the Madison River, mussels remain sparse and non-viable (Stagliano 2008, unpublished data). The declining status of the WEPE has led to its designation as a Tier 1 Species of Conservation Need (SGCN) by MT Fish Wildlife and Parks (2006), a Species of Concern by the State of Montana (MTNHP 2008) and a Sensitive Species by the U.S. Forest Service Northern Region 1 (2011, S. Spaulding pers. comm.).

The best approach to conserving aquatic species is to ensure that habitats are managed in ways that maintain healthy ecosystems and allow the full complement of native species to flourish. Turner Enterprises has come a long way to achieve this goal on the Flying D Ranch with the restoration of Cherry Creek to a native westslope cutthroat trout stream. To fully succeed in the goal of harboring all native aquatic species, the western pearlshell mussel, found naturally below the natural barrier but prefers the westslope cutthroat trout as its native host fish, needs to be included in the Cherry Creek restoration plan.

Objectives. This project will: 1) evaluate the feasibility of translocating the WEPE into Cherry Creek above the natural fish barrier on the Flying D Ranch by evaluating habitat suitability throughout the $\sim 10$ mile reach of Cherry Creek in the context of stream habitat parameters known to contain populations of WEPE in Montana, 2) Measure and identify stream geomorphology parameters known to be favored by WEPE including stable stream reaches with point bars, willow/alder dominated riparian, minimal bedload movement and a Rosgen C3-C4 stream classification (i.e. gradient $<2 \%,>1.2$ sinuosity, $>12$ width/depth ratio, avg. substrate size 32-45mm) (Rosgen 1996), 3) Identify a suitable donor population in the Madison River 
watershed and have 30 specimens from that population tested by the FWP Fish Health Committee (following MTFWP 2010 protocols). If all objectives are met, we would be positioned to begin translocations in 2013 given positive habitat feasibility ranks and a certified disease-free, donor population.

Methods. We used widely accepted Rosgen Level II methodology to evaluate, quantitatively measure and analyze stream geomorphology on Flying D Ranch Cherry Creek sections (Rosgen 1996). Parameters such as channel gradient and stability, point bar formation and sinuosity were measured by walking the reach at the high water mark and stretching a chain-man line as a distance measure, while a straight line measure was calculated from GPS readings taken at the top and bottom of the reach using a Garmin 60 CSX; the ratio of chain-man distance to straight line measures is the calculated sinuosity. Gradient was calculated with the laser level and stadium rod as the difference between stadium height reading at the wetted edge of the laser location minus the stage height reading at a distance of at least $20 x$ wetted widths downstream divided by that reach distance. Channel cross sectional data was measured using a laser level at the crest of the riffles where the pebble count (100 particles counted) was performed. This data was entered on standardized Laser Level Channel Cross-Section, Pebble Count and Stream Classification forms to provide the necessary variables to assign a Rosgen Stream Classification. We then ranked suitability for WEPE translocation based on the stream classification, with reaches classified as C4 ranking highest.

We also searched database records for previous mussel surveys in the Madison River basin and performed additional mussel surveys on streams that had few to no survey records in the basin. We used visual encounter surveys standardized for the statewide mussel surveys protocols (Stagliano 2010).

\section{Results}

We performed Rosgen Level II evaluations on ten Cherry Creek stream reach sections, seven above the natural barrier in the Butler Reach and three above the man-made barrier (Table 1, Map 1 and 2). Stream reach sites behind and upstream of the ranch house (sites \#825-828) and 
just upstream of the natural barrier canyon (site \#832) have limited potential as WEPE translocation sites due to the higher gradient and larger substrate classes (Rosgen B3) as indicated in their pebble counts (Table 1, Appendix A). The most suitable WEPE translocation sites are within the Butler Reach (4 sites) and one reach above the man-made barrier (\#823) (Table 2, Map 1 \& 2).

Table 1. Rosgen II field measurements used in stream classification. Highest ranking preferred WEPE habitat site are bolded and shaded.

\begin{tabular}{|c|c|c|c|c|c|c|c|c|}
\hline $\begin{array}{l}\text { Site } \\
\text { ID }\end{array}$ & $\begin{array}{c}\text { Bankfull } \\
\text { Channel } \\
\text { Width } \\
\text { (m) }\end{array}$ & $\begin{array}{c}\text { Entrench } \\
\text { ment } \\
\text { Ratio }\end{array}$ & $\begin{array}{l}\text { Width / } \\
\text { Depth } \\
\text { Ratio }\end{array}$ & $\begin{array}{l}\text { Estimated } \\
\text { Sinuosity }\end{array}$ & $\begin{array}{c}\text { Stream } \\
\text { Slope } \\
(\%)\end{array}$ & $\begin{array}{c}\text { Riffle } \\
\text { Pebble } \\
\text { Count } \\
\text { D50 }\end{array}$ & $\begin{array}{c}\text { Rosgen } \\
\text { Stream } \\
\text { Type }\end{array}$ & $\begin{array}{c}\text { Suitability } \\
\text { Rank }\end{array}$ \\
\hline 116 & 6.7 & 2.1 & 24.0 & 1.20 & 1.9 & $90-128$ & B3/C3b & \\
\hline 119 & 6.9 & 2.8 & 25.4 & 1.67 & 0.9 & $45-64$ & $\mathrm{C} 4 \mathrm{~b}$ & $5^{\text {th }}$ \\
\hline 819 & 7.0 & 2.2 & 26.0 & 1.50 & 1.1 & $64-90$ & c3b & $5^{\text {th }}$ \\
\hline 821 & 9.7 & 2.5 & 31.5 & 1.90 & 0.6 & $64-90$ & C3 & $4^{\text {th }}$ \\
\hline 823 & 11.4 & 2.3 & 34.0 & 1.14 & 0.5 & $32-45$ & C4 & $3^{\text {rd }}$ \\
\hline 825 & 6.6 & 2.2 & 20.1 & 1.20 & 1.7 & $90-128$ & B3/C3b & \\
\hline 826 & na & na & na & na & na & na & na & \\
\hline 827 & 10.1 & 8.0 & 32.2 & 1.20 & 1.5 & $90-128$ & C3 & \\
\hline 829 & 8.8 & 2.1 & 22.0 & 1.20 & 1.8 & $90-128$ & $\mathrm{~B} 3 / \mathrm{C} 3 \mathrm{~b}$ & \\
\hline 830 & 10.1 & 4.1 & 18.7 & 1.35 & 0.8 & $64-90$ & C4/C3 & $2^{\text {nd }}$ \\
\hline 831 & 10.1 & 4.1 & 18.7 & 1.35 & 0.8 & $64-90$ & C4/C3 & $2^{\text {nd }}$ \\
\hline 832 & 9.5 & 1.8 & 14.0 & 1.10 & 2.1 & $90-128$ & B3 & \\
\hline 833 & 10.2 & 2.9 & 29.0 & 1.24 & 0.7 & $45-64$ & C4 & $1^{\text {st }}$ \\
\hline 834 & 10.2 & 2.9 & 29.0 & 1.24 & 0.7 & $45-64$ & C4 & $1^{\text {st }}$ \\
\hline
\end{tabular}


Table 2. Cherry Creek and Spanish Creek sites visited during the TEl Ranch Surveys 2012. Stream sites are organized from higher elevation --> downstream. Shaded sites are those ranked most suitable for WEPE translocations. TDS $=$ Total Dissolved Solids

\begin{tabular}{|c|c|c|c|c|c|c|c|c|c|c|c|c|}
\hline \begin{tabular}{c|} 
Site \\
Number
\end{tabular} & Site Code & latitude & longitude & Date/time & $\begin{array}{c}\text { Elevation } \\
(\mathrm{m})\end{array}$ & $\begin{array}{c}\text { Rosgen } \\
\text { Class }\end{array}$ & Site Description & $\begin{array}{c}\text { Temp } \\
{ }^{\circ} \mathrm{C}\end{array}$ & $\begin{array}{c}\text { Cond } \\
(\mu \mathrm{s} / \mathrm{sec})\end{array}$ & $\mathrm{pH}$ & TDS & Photo Link \\
\hline 829 & UM_CC829 & 45.5157 & -111.4478 & 7/24/2012 7:37 & 1742 & $\mathrm{~B} 3 / \mathrm{C} 3$ & $\begin{array}{l}\text { Cherry Creek above the ranch reach, boulders with } \\
\text { some embedded cobbles trapping sand }\end{array}$ & 12.1 & 37 & 7.8 & 18 & $\begin{array}{l}\text { http://mtnhp.org/ThumbsPlus/Eco/Aquati } \\
\text { MusselSurveys/UM CC829.JPG }\end{array}$ \\
\hline 827 & UM_CC827 & 45.5188 & -111.4453 & 7/24/2012 7:21 & 1735 & C3 & $\begin{array}{l}\text { Cherry Creek above the active beaver pond, B3/C3 } \\
\text { Rosgen reach boulder dominated }\end{array}$ & 12.2 & 39 & 7.7 & 19 & $\begin{array}{l}\text { http://mtnhp.org/ThumbsPlus/Eco/Aquatics/ } \\
\text { MusselSurveys/UM_CC827.JPG }\end{array}$ \\
\hline 826 & UM_CC826 & 45.5222 & -111.4478 & 7/24/2012 7:12 & 1731 & na & $\begin{array}{l}\text { Cherry Creek behind the cow camp with an active } \\
\text { beaver pond }\end{array}$ & 12.7 & 38 & 7.7 & 20 & $\begin{array}{l}\text { http://mtnhp.org/ThumbsPlus/Eco/Aquatics/ } \\
\text { MusselSurveys/UM CC826 bp.JPG }\end{array}$ \\
\hline 825 & UM_CC825 & 45.5226 & -111.4459 & 7/24/2012 7:09 & 1706 & B3/C3b & Cherry Creek behind the cow camp & 12.3 & 37 & 7.7 & 19 & $\begin{array}{l}\text { http://mtnhp.org/ThumbsPlus/Eco/Aquatics/ } \\
\text { MusselSurveys/UM_CC825.JPG }\end{array}$ \\
\hline 823 & UM_CC823 & 45.5508 & -111.4466 & $7 / 23 / 2012$ 19:30 & 1674 & C4 & Cherry Creek above the man-made & 11.7 & 126 & 8.1 & 62 & $\begin{array}{l}\text { http://mtnhp.org/ThumbsPlus/Eco/Aquatics/ } \\
\text { MusselSurveys/UM CC823 ov.JPG }\end{array}$ \\
\hline 116 & UM_CC116 & 45.5512 & -111.4460 & 7/23/2012 14:02: & 1672 & $\mathrm{~B} 3 / \mathrm{C} 3 \mathrm{~b}$ & $\begin{array}{l}\text { Cherry Creek upstream of site } 819 \text { at upper end of } \\
\text { Bulter Reach }\end{array}$ & 12.5 & 122 & 8.1 & 60 & $\begin{array}{l}\text { http://mtnhp.org/ThumbsPlus/Eco/Aquatics/ } \\
\text { MusselSurveys/UM CC116.JPG }\end{array}$ \\
\hline 819 & UM_CC819 & 45.5542 & -111.4443 & $7 / 23 / 201212: 54$ & 1671 & C3b & $\begin{array}{l}\text { Cherry Creek on the Turner Ranch upper end of } \\
\text { Butler Reach } \\
\text { Cherry Creek on the Turner Ranch upper end of }\end{array}$ & 2.5 & 4 & 8.1 & 62 & $\begin{array}{l}\text { http://mtnhp.org/ThumbsPlus/Eco/Aquatics/ } \\
\text { MusselSurveys/UM CC821 t.JPG } \\
\text { http://mtnhp.org/ThumbsPlus/Eco/Aquatics/ }\end{array}$ \\
\hline 821 & UM_CC821 & 45.5579 & -111.4446 & $7 / 23 / 201214: 54$ & 1670 & c3b & $\begin{array}{l}\text { Butler Reach } \\
\text { Cherry Creek upstream of site } 833 \text { at lower end of } \\
\text { Bulter Reach }\end{array}$ & 12.9 & 129 & 8.2 & 62 & $\begin{array}{l}\text { Mussel_Surveys/UM_C } \\
\text { http://mtnhp.org/Thur } \\
\text { MusselSurveys/UM_C }\end{array}$ \\
\hline 833 & UM_CC833 & 45.5652 & -111.4419 & $7 / 24 / 201210: 53$ & 1665 & $\mathrm{C} 3 / \mathrm{C} 4$ & $\begin{array}{l}\text { Cherry Creek in the Butler meadow reach } \\
\text { transistioning from a } \mathrm{C} 3 \text { to a } \mathrm{C} 4 \\
\text { Cherry Creek in the Butler meadow reach, great C4 }\end{array}$ & 12.2 & 119 & 8.1 & 62 & $\begin{array}{l}\text { http://mtnhp.org/ThumbsPlus/Eco/Aquatics/ } \\
\text { MusselSurveys/UM_CC833.JPG } \\
\text { http://mtnhp.org/ThumbsPlus/Eco/Aquatics/ }\end{array}$ \\
\hline 834 & UM_CC834 & 45.5642 & -111.4417 & $7 / 24 / 201211: 06$ & 1664 & C4 & $\begin{array}{l}\text { WEPE habitat } \\
\text { Cherry Creek in the lower butler meadow reach }\end{array}$ & 12.2 & 119 & 8.1 & 62 & $\begin{array}{l}\text { MusselSurvevs/UM_CC833_u.JPG } \\
\text { http://mtnhp.org/ThumbsPlus/Eco/Aquatics/ }\end{array}$ \\
\hline 830 & UM_CC830 & 45.5659 & -111.4405 & $7 / 24 / 20129: 22$ & 1663 & $\mathrm{C} 3 / \mathrm{C} 4$ & $\begin{array}{l}\text { transistioning from a C3 to a B3 } \\
\text { Cherry Creek in the lower butler meadow reach }\end{array}$ & 11.5 & 115 & 8.2 & 57 & $\begin{array}{l}\text { MusselSurveys/UM CC830.JPG } \\
\text { http://mtnhp.org/ThumbsPlus/Eco/Aquatics/ }\end{array}$ \\
\hline 831 & UM_CC831 & 45.5654 & -111.4409 & $7 / 24 / 20129: 28$ & 16 & $\mathrm{C} 3 / \mathrm{C} 4$ & transistioning from a $\mathrm{C} 3$ to $\mathrm{a} B 3$ & & & 8.2 & 57 & Mussels \\
\hline 832 & UM_CC832 & 45.5674 & -111.4391 & 7/24/2012 10:31 & 1660 & B3 & $\begin{array}{l}\text { Cherry Creek exiting the Butler reach transistioning } \\
\text { from a C3 to a B3 }\end{array}$ & & & 8.1 & 62 & $\begin{array}{l}\text { http://mtnhp.org/ThumbsPlus/Eco/Aquatics/ } \\
\text { Mussel_Surveys/UM_CC832.JPG }\end{array}$ \\
\hline 835 & UM_CC835 & 45.5947 & -111.4907 & 7/24/2012 12:13 & 1388 & C3 & $\begin{array}{l}\text { Cherry Creek below the natural barrier transistioning } \\
\text { from a C3 to a B } 3\end{array}$ & 6 & & 8.5 & 70 & $\begin{array}{l}\text { http://mtnhp.org/ThumbsPlus/Eco/Aquatics/ } \\
\text { MusselSurveys/UM_CC835.JPG }\end{array}$ \\
\hline 836 & UM_CC836 & 45.5938 & -111.4890 & $7 / 24 / 201212: 33$ & 1386 & B3 & $\begin{array}{l}\text { Cherry Creek below the natural barrier transistioning } \\
\text { from a C3 to a B3 }\end{array}$ & 6 & 141 & 8.5 & 70 & $\begin{array}{l}\text { http://mtnhp.org/ThumbsPlus/Eco/Aquatics/ } \\
\text { MusselSurveys/UM_cc836_d.JPG }\end{array}$ \\
\hline 816 & UM_CC816 & 45.6172 & -111.5432 & 7/23/2012 10:13 & 1363 & -4 & Cherry Creek below the county road bridge off Ranch & 17.8 & 165 & 8.0 & 82 & $\begin{array}{l}\text { http://mtnhp.org/ThumbsPlus/Eco/Aquatics/ } \\
\text { MusselSurveys/UM CC816.JPG }\end{array}$ \\
\hline 837 & UM_SPC837 & 45 & -1 & 214:10 & 22 & B3 & Spanish Creek on $\mathrm{t}$ & 16.3 & 3 & 7.9 & 22 & $\begin{array}{l}\text { http://mtnhp.org/ThumbsPlus/Eco/Aquatics/ } \\
\text { Musselsurveys/UM_SPC837.JPG } \\
\text { http://mtnhp.org/InumbsPIus/tco/Aquatics/ }\end{array}$ \\
\hline 838 & UM_SPC838 & 45.4986 & -111.3240 & $7 / 24 / 201214: 30$ & 1668 & C3 & Spanish Creek on the TEI Property & 16.5 & 44 & 7.9 & 24 & MusselSurveys/UM SPC838.JPG \\
\hline 839 & UM_SPC839 & 45.4976 & -111.3251 & 7/24/2012 14:43 & 1665 & C3 & Spanish Creek on the TEI Property & 16.5 & 44 & 7.9 & 22 & $\begin{array}{l}\text { http://mtnhp.org/ThumbsPlus/Eco/A } \\
\text { MusselSurveys/UM SPC839.JPG }\end{array}$ \\
\hline
\end{tabular}


Map 1. Cherry Creek on TEI Property from manmade barrier (site 115) downstream into Butler Reach

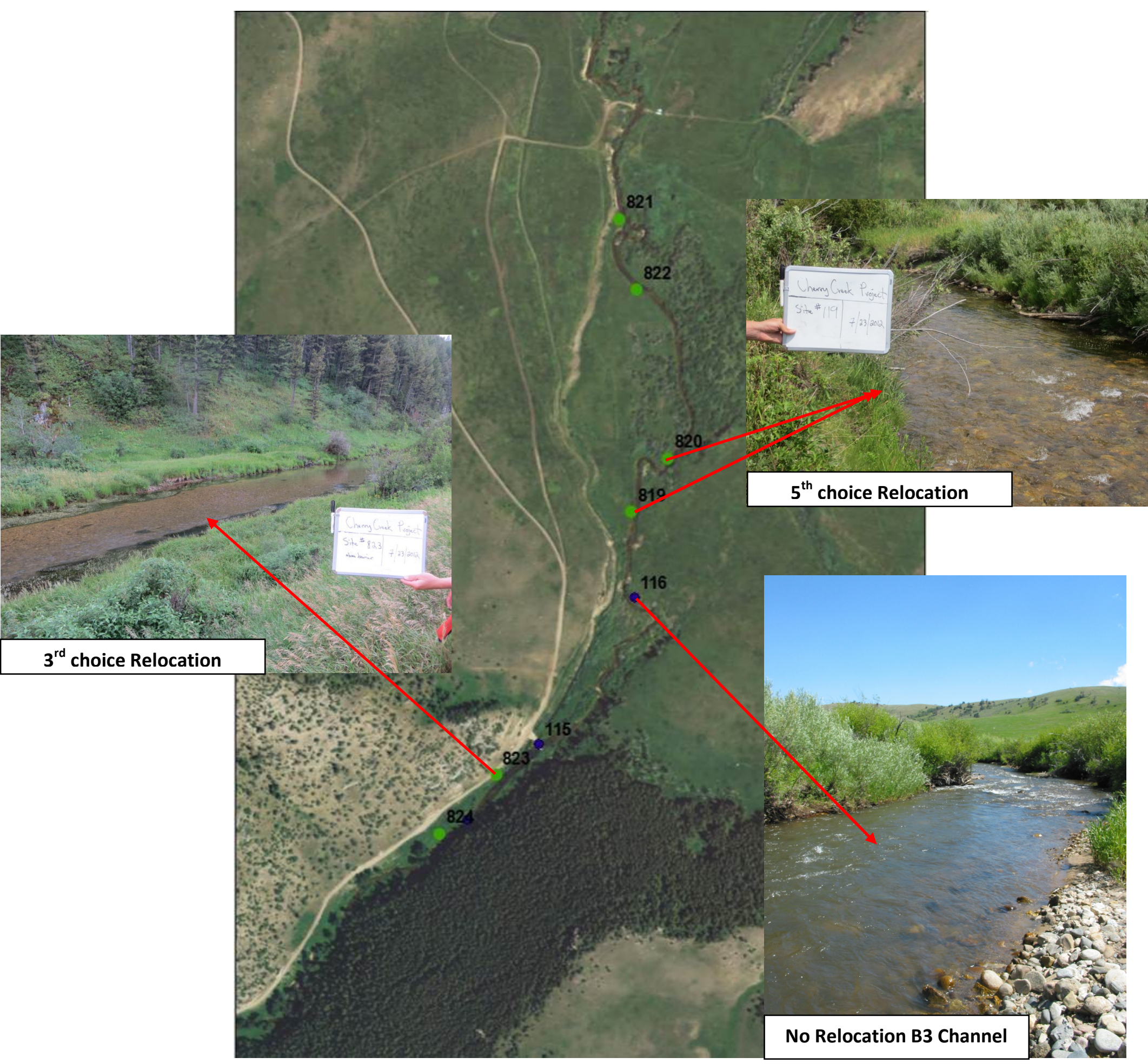


Map 2. Cherry Creek continued from Map 1 (site 820) downstream thru Butler Reach to the canyon (Site 832)

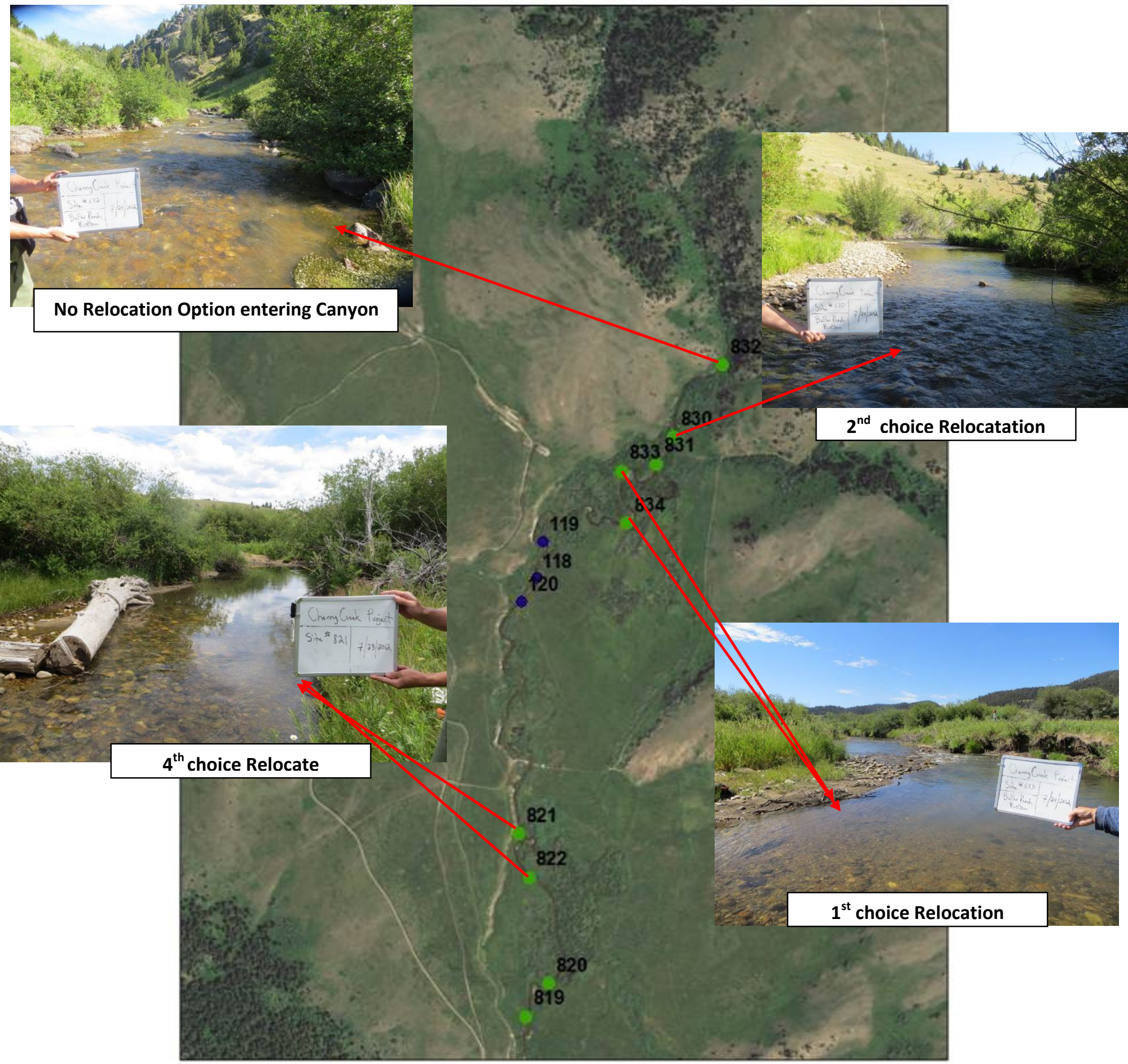


We surveyed 25 previously unvisited stream reaches in 11 streams within the Madison and Jefferson River Basins during the summer of 2012 trying to locate a donor WEPE population (Map 3). Despite considerable survey effort, we did not find a single live WEPE mussel. We identified recently dead WEPE shells in a new reach of O'Dell Spring Creek indicating the

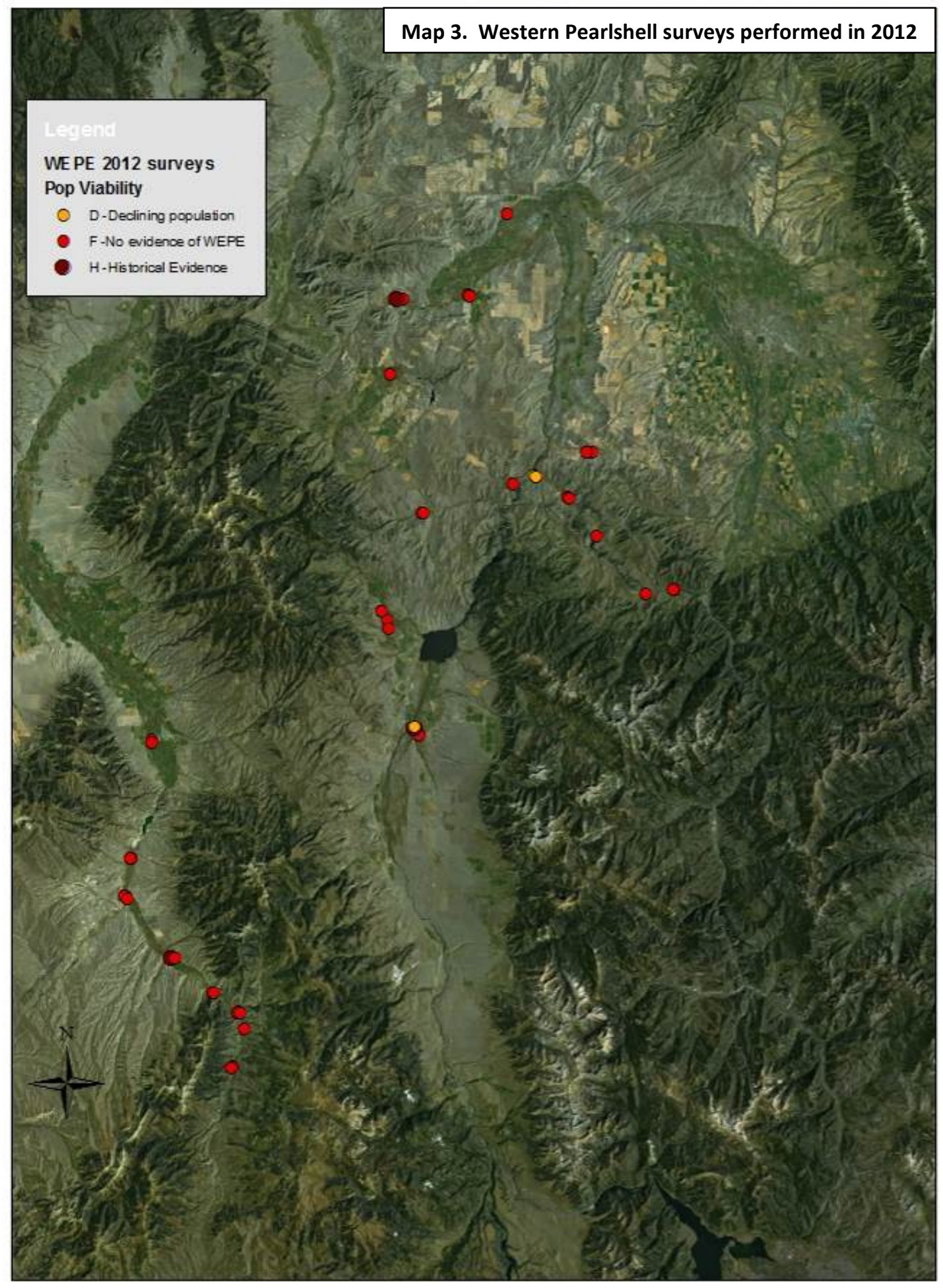


potential of a live population (D-rank viability), but the two other streams containing WEPE shells, the Ruby and Jefferson Rivers, only had relic shells (>10 years old)and are not likely to contain live populations. Of fifty-one stream reaches in the Madison River basin that had been surveyed for WEPE prior to 2012 (Appendix B), only one of those reaches was ranked as having a viable WEPE population (Duck Creek, B-rank)(Map 4). We did not return to Duck Creek in 2012 to collect mussels for the fish health screening, but plan to revisit this site in 2013 to

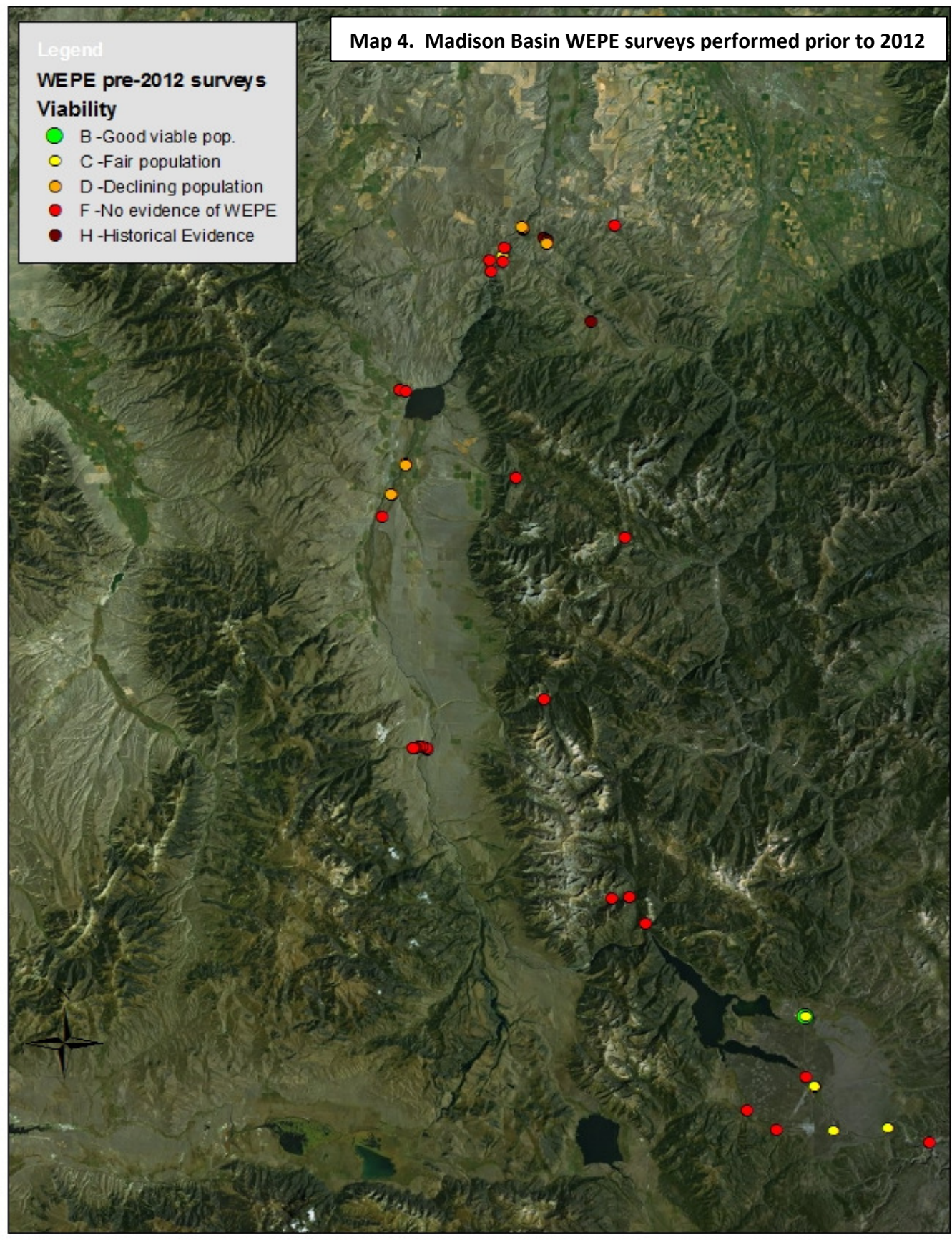


evaluate Whirling Disease Status with a backpack shocking grab sample of rainbow trout and a WEPE collection to submit for health screen analysis as required for translocation (FWP 2010). Previous whirling disease testing on 136 total trout from Duck Creek in 2004, 2005 and 2007 showed 0\% infected, except for 1 outlier in 2007 (1 out of 43 infected at a Grade 5)(P. Clancy, pers. comm.). Pat said "When they got a real outlier like this they attributed it to a fish getting mixed into the wrong aquarium at Pony (they had occasional problems with jumpers), a mix up in processing prior to sending to the lab, or a mix-up at the lab." Thus, Duck Creek could be considered WD- and our Madison River basin donor WEPE population with an additional WD negative trout sample and a clean Fish Health Mussel Screen in 2013.

\section{Conclusion}

Completion of extensive habitat analysis within the Cherry Creek Native Trout Project Reach, allows us to have the scientific evidence along with our professional opinion to confidently determine that at least five reaches of Cherry Creek on the Turner Flying D Ranch contain the preferred stream habitat, as well as suitable densities of their native host fish (WSCT) capable for allowing the western pearlshell mussel to survive, reproduce, and persist once translocated to this stream. As evidenced by our 2012 and previous surveys, WEPE populations are in decline throughout the Madison River basin and another secure (viable) population in Cherry Creek would considerably aid conservation efforts for this species.

\section{Literature Cited}

Montana Fish, Wildlife and Parks 2010. Environmental Assessement: Western pearlshell mussel translocation project, Blackfoot River Basin, MT. Preparers: R. Pierce, L. Knotek and D. Stagliano. http://fwpiis.mt.gov/content/getltem.aspx?id=43287

Rosgen, D. 1996. Applied river morphology manual. Wildlands Hydrology, Pagosa Springs, CO

Stagliano, D.M. and R.G. Pierce 2011. Western Pearlshell Mussel Translocation Project in the Blackfoot River Watershed: How not to treat mussels prior to relocation. Presented at the 44th Annual MT Chapter AFS Meeting, Great Falls, MT

Stagliano, D. 2010. Freshwater Mussels in Montana: Comprehensive Results from 3 years of SWG Funded Surveys. Report to MT Fish Wildlife and Parks, Helena, MT 41 pp. + appendices 


\begin{tabular}{|c|c|c|c|c|c|c|c|c|c|c|c|c|c|c|c|}
\hline Reach ID & $\begin{array}{c}\text { Pebble } \\
\text { Count } \\
\text { (bkf } \\
\text { width m) }\end{array}$ & $\begin{array}{c}\text { Riffle } \\
\text { Pebble } \\
\text { Count } \\
\text { D50 }\end{array}$ & $<2 m m$ & $\begin{array}{l}2-4 \\
m m\end{array}$ & $\begin{array}{l}4.1- \\
5.7 \\
\mathrm{~mm}\end{array}$ & $\begin{array}{c}5.7-8 \\
\mathrm{~mm}\end{array}$ & $\begin{array}{c}8- \\
11.3 \\
m m\end{array}$ & $\begin{array}{c}11.4- \\
16 \\
\mathrm{~mm}\end{array}$ & $\begin{array}{c}16- \\
22.6 \\
\mathrm{~mm}\end{array}$ & $\begin{array}{c}22.6- \\
32 \mathrm{~mm}\end{array}$ & $\begin{array}{c}32-45 \\
\mathrm{~mm}\end{array}$ & $\begin{array}{c}45- \\
64 \\
m m\end{array}$ & $\begin{array}{c}64-90 \\
\mathrm{~mm}\end{array}$ & $\begin{array}{l}90- \\
128 \\
\mathrm{~mm}\end{array}$ & $>128 \mathrm{~mm}$ \\
\hline 116 & 6.8 & $90-128$ & 6 & 4 & 3 & 3 & 2 & 2 & 6 & 7 & 10 & 10 & 17 & 15 & 15 \\
\hline 119 & 6.8 & $45-64$ & 14 & 5 & 2 & 2 & 3 & 3 & 7 & 8 & 17 & 9 & 13 & 7 & 10 \\
\hline 819 & 7.0 & $64-90$ & 9 & 8 & 4 & 5 & 6 & 5 & 2 & 6 & 9 & 11 & 8 & 11 & 16 \\
\hline 821 & 9.6 & $64-90$ & 9 & 8 & 4 & 5 & 6 & 5 & 2 & 6 & 9 & 11 & 8 & 11 & 16 \\
\hline 823 & 11.0 & $32-45$ & 18 & 3 & 4 & 2 & 2 & 3 & 9 & 13 & 20 & 17 & 5 & 2 & 2 \\
\hline 825 & 6.7 & $90-128$ & 6 & 8 & 4 & 5 & 6 & 5 & 2 & 6 & 9 & 11 & 8 & 14 & 16 \\
\hline 826 & na & na & na & na & na & na & na & na & na & na & na & na & na & na & na \\
\hline 827 & 9.0 & $90-128$ & 6 & 7 & 2 & 5 & 5 & 2 & 6 & 9 & 5 & 8 & 9 & 9 & 27 \\
\hline 829 & 9.8 & $90-128$ & 6 & 7 & 2 & 5 & 5 & 2 & 6 & 8 & 5 & 8 & 9 & 12 & 25 \\
\hline 830 & 10.1 & $64-90$ & 8 & 8 & 4 & 1 & 3 & 4 & 4 & 3 & 10 & 16 & 9 & 18 & 12 \\
\hline 831 & 10.1 & $64-90$ & 8 & 8 & 4 & 1 & 3 & 4 & 4 & 3 & 10 & 16 & 9 & 18 & 12 \\
\hline 832 & 10.1 & $90-128$ & 6 & 4 & 4 & 1 & 3 & 4 & 4 & 3 & 7 & 10 & 17 & 15 & 22 \\
\hline 833 & 10.2 & $45-60$ & 8 & 7 & 7 & 4 & 1 & 5 & 4 & 7 & 11 & 17 & 9 & 10 & 10 \\
\hline 834 & 10.2 & $45-60$ & 8 & 7 & 7 & 4 & 1 & 5 & 4 & 7 & 11 & 17 & 9 & 10 & 10 \\
\hline
\end{tabular}


Appendix B. WEPE Surveys performed in the Madison River Basin prior to 2012. Viability Code: B-good viability, C -fair population, D -declining, not viable, F- absent, failed to find evidence of a population, $\mathrm{H}$-Historical Verified Site, population now absent)

\begin{tabular}{|c|c|c|c|c|c|}
\hline Stream & Latitude & Longitude & Mussel_species & Viability & $\begin{array}{c}\text { Observation } \\
\text { Date }\end{array}$ \\
\hline Cabin Creek & 44.87640 & -111.34560 & No Mussels Found & $\mathrm{F}$ & 29-Jul-07 \\
\hline Cherry Creek & 45.61977 & -111.54643 & No Mussels Found & $\mathrm{F}$ & 14-Jul-08 \\
\hline Cherry Creek & 45.62191 & -111.54842 & Margaritifera falcata & $\mathrm{D}$ & 15-Jul-08 \\
\hline Cherry Creek & 45.62143 & -111.54659 & Margaritifera falcata & $\mathrm{D}$ & 15-Jul-08 \\
\hline Cherry Creek & 45.62285 & -111.54975 & Margaritifera falcata & D & 15-Jul-08 \\
\hline Cherry Creek & 45.61252 & -111.51614 & $\begin{array}{l}\text { Margaritifera falcata } \\
\text { shells, no live }\end{array}$ & $\mathrm{H}$ & 26-Aug-10 \\
\hline Cherry Creek & 45.61051 & -111.51238 & $\begin{array}{l}\text { Margaritifera falcata } \\
\text { shells, no live }\end{array}$ & $\mathrm{H}$ & 26-Aug-10 \\
\hline Cherry Creek & 45.60902 & -111.51261 & $\begin{array}{l}\text { Margaritifera falcata } \\
\text { shells, no live }\end{array}$ & $\mathrm{H}$ & 26-Aug-10 \\
\hline Cherry Creek & 45.60630 & -111.51176 & Margaritifera falcata & $\mathrm{D}$ & 26-Aug-10 \\
\hline Cherry Creek & 45.52255 & -111.44596 & No Mussels Found & $\mathrm{H}$ & 26-Aug-10 \\
\hline Cougar Creek & 44.71508 & -111.11069 & No Mussels Found & $\mathrm{F}$ & 31-Jul-07 \\
\hline Duck Creek & 44.77958 & -111.11499 & Margaritifera falcata & C & 31-Jul-07 \\
\hline Duck Creek & 44.77998 & -111.11357 & No Mussels Found & $\mathrm{F}$ & 31-Jul-07 \\
\hline Duck Creek & 44.77956 & -111.11249 & Margaritifera falcata & B & 16-Jul-08 \\
\hline Elk Creek & 45.62670 & -111.41430 & No Mussels Found & $\mathrm{F}$ & 15-Jul-07 \\
\hline Fourmile Creek & 45.34124 & -110.23215 & No Mussels Found & $\mathrm{F}$ & 15-Jul-07 \\
\hline Hot Springs Creek & 45.58564 & -111.59409 & No Mussels Found & $\mathrm{F}$ & $15-J u l-08$ \\
\hline Jack Creek & 45.35338 & -111.54909 & No Mussels Found & $\mathrm{F}$ & 15-Jul-07 \\
\hline Madison River & 44.64702 & -110.93216 & No Mussels Found & $\mathrm{F}$ & 31-Jul-07 \\
\hline Madison River & 44.66194 & -110.99187 & Margaritifera falcata & C & 31-Jul-07 \\
\hline Madison River & 44.70339 & -111.09805 & Margaritifera falcata & C & 31-Jul-07 \\
\hline Madison River & 44.70484 & -111.09695 & Margaritifera falcata & C & 31-Jul-07 \\
\hline Madison River & 44.65740 & -111.06970 & Margaritifera falcata & $\mathrm{C}$ & 31-Jul-07 \\
\hline Madison River & 44.77988 & -111.11298 & Margaritifera falcata & C & 16-Jul-08 \\
\hline Madison River & 45.59050 & -111.57620 & Margaritifera falcata & C & 13-Jul-02 \\
\hline Madison River & 45.58510 & -111.57629 & No Mussels Found & $\mathrm{F}$ & 15-Jul-08 \\
\hline Madison River & 45.57462 & -111.59357 & No Mussels Found & $\mathrm{F}$ & 16-Jul-08 \\
\hline $\begin{array}{l}\text { Madison River } \\
\text { side channel }\end{array}$ & 45.59978 & -111.57332 & No Mussels Found & $\mathrm{F}$ & 15-Jul-08 \\
\hline Meadow Creek & 45.44380 & -111.71891 & No Mussels Found & $\mathrm{F}$ & 15-Jul-08 \\
\hline
\end{tabular}




\begin{tabular}{|l|l|l|l|l|l|}
\hline Meadow Creek & 45.44259 & -111.71032 & No Mussels Found & F & 15-Jul-08 \\
\hline $\begin{array}{l}\text { Middle Fork of } \\
\text { West Fork }\end{array}$ & 45.29219 & -111.38807 & No Mussels Found & F & 07-Aug-07 \\
\hline No Man Creek & 45.11550 & -111.49810 & No Mussels Found & F & 17-Aug-05 \\
\hline $\begin{array}{l}\text { North Fork } \\
\text { Meadow Creek }\end{array}$ & 45.65757 & -111.89389 & No Mussels Found & F & 14-Jul-08 \\
\hline O'dell Creek & 45.36590 & -111.70720 & No Mussels Found & F & 02-Aug-11 \\
\hline O'dell Creek & 45.36514 & -111.70708 & Margaritifera falcata & D & 02-Aug-11 \\
\hline O'dell Creek & 45.36378 & -111.70731 & Margaritifera falcata & D & 02-Aug-11 \\
\hline O'dell Creek & 45.33248 & -111.72650 & Margaritifera falcata & D & 02-Aug-11 \\
\hline O'dell Creek & 45.33113 & -111.72831 & Margaritifera falcata & D & 02-Aug-11 \\
\hline $\begin{array}{l}\text { O'dell Spring } \\
\text { Creek }\end{array}$ & 45.30711 & -111.73920 & No Mussels Found & F & 15-Jul-07 \\
\hline Ruby Creek & 45.05903 & -111.66366 & No Mussels Found & F & 01-Aug-11 \\
\hline Ruby Creek & 45.05957 & -111.66560 & No Mussels Found & F & 01-Aug-11 \\
\hline Ruby Creek & 45.06073 & -111.67164 & No Mussels Found & F & 01-Aug-11 \\
\hline Ruby Creek & 45.06114 & -111.67307 & No Mussels Found & F & 01-Aug-11 \\
\hline Ruby Creek & 45.06183 & -111.67633 & No Mussels Found & F & 02-Aug-11 \\
\hline Ruby Creek & 45.06051 & -111.68027 & No Mussels Found & F & 02-Aug-11 \\
\hline Ruby Creek & 45.06013 & -111.68352 & No Mussels Found & F & 02-Aug-11 \\
\hline Ruby Creek & 45.06020 & -111.68547 & No Mussels Found & F & 02-Aug-11 \\
\hline $\begin{array}{l}\text { South Fork } \\
\text { Madison River }\end{array}$ & 44.65752 & -111.15062 & No Mussels Found & F & 31-Jul-07 \\
\hline $\begin{array}{l}\text { South Fork } \\
\text { Madison River }\end{array}$ & 44.67870 & -111.19432 & No Mussels Found & F & 31-Jul-07 \\
\hline $\begin{array}{l}\text { West Fork Beaver } \\
\text { Creek }\end{array}$ & 44.90506 & -111.36955 & No Mussels Found & F & 29-Jul-07 \\
\hline Creek trib & 44.90346 & -111.39455 & No Mussels Found & F & 29-Jul-07 \\
\hline & & & & & \\
\hline
\end{tabular}

